PROCEEDINGS OF THE

AMERICAN MATHEMATICAL SOCIETY

Volume 132, Number 2, Pages 503-512

S 0002-9939(03)07063-1

Article electronically published on July 2, 2003

\title{
SEQUENTIAL PRODUCT OF QUANTUM EFFECTS
}

\author{
AURELIAN GHEONDEA AND STANLEY GUDDER
}

(Communicated by Joseph A. Ball)

\begin{abstract}
Unsharp quantum measurements can be modelled by means of the class $\mathcal{E}(\mathcal{H})$ of positive contractions on a Hilbert space $\mathcal{H}$, in brief, quantum effects. For $A, B \in \mathcal{E}(\mathcal{H})$ the operation of sequential product $A \circ B=A^{1 / 2} B A^{1 / 2}$ was proposed as a model for sequential quantum measurements. We continue these investigations on sequential product and answer positively the following question: the assumption $A \circ B \geq B$ implies $A B=B A=B$.

Then we propose a geometric approach of quantum effects and their sequential product by means of contractively contained Hilbert spaces and operator ranges. This framework leads us naturally to consider lattice properties of quantum effects, sums and intersections, and to prove that the sequential product is left distributive with respect to the intersection.
\end{abstract}

\section{INTRODUCTION}

Unsharp quantum measurements experiments can be modelled by means of the class $\mathcal{E}(\mathcal{H})$ of positive contractions on a Hilbert space $\mathcal{H}$. For $A, B \in \mathcal{E}(\mathcal{H})$ the operation of sequential product $A \circ B=A^{1 / 2} B A^{1 / 2}$ was proposed as a model for sequential measurements. A careful investigation of properties of the sequential product has been carried over in [5] (see also [6]). In that paper it was proved that, if the underlying Hilbert space $\mathcal{H}$ is finite dimensional, then, by preceding the effect $B$ with another effect $A$, we cannot amplify the effect $B$. In terms of the operator model, this means that if for some $A, B \in \mathcal{E}(\mathcal{H})$ we have $A \circ B \geq B$, then $A B=B A=B$. The question, raised in [5], is whether this is true for infinite-dimensional Hilbert spaces as well. We answer positively this question in Theorem [2.6. The idea of our proof is to iterate the inequality $A \circ B \geq B$ and to show that, at the limit, we obtain exactly the condition $A B=B A=B$. Here we use the borelian functional calculus for selfadjoint operators, that is, we pass to the von Neumann algebra generated by the operator $A$ to get at the limit the orthogonal projection onto $\operatorname{Ker}(I-A)$, a common procedure in ergodic theory. The second section also contains a few other results on the sequential product, especially concerning comparison.

In the third section we propose a geometric approach of the sequential product based on the interpretation of quantum effects as contractively contained Hilbert spaces. This is a particular situation of the theory of continuously contained Hilbert spaces in quasi-complete locally convex spaces of L. Schwartz [8]. Similar ideas have

Received by the editors August 29, 2002 and, in revised form, October 17, 2002.

2000 Mathematics Subject Classification. Primary 47B65, 81P15, 47N50, 46C07. 
been used in the case of contractively contained Hilbert spaces in the de BrangesRovnyak model in quantum scattering [2], and operator ranges; cf. [4] and [7]. This allows us to consider lattice properties, as sums and intersections of the set of quantum effects, that lead naturally to the parallel sum of quantum effects. The sequential product is a morphism, in the second variable, with respect to the binary operation of parallel sum; cf. Theorem 3.10 Rephrased in terms of contractively contained Hilbert spaces, this means that the sequential product is left distributive with respect to the intersection.

\section{Sequential product}

Let $\mathcal{H}$ be a Hilbert space. We denote by $\mathcal{B}(\mathcal{H})$ the $C^{*}$-algebra of linear and bounded operators on $\mathcal{H}, \mathcal{B}(\mathcal{H})^{+}=\{A \in \mathcal{B}(\mathcal{H}) \mid A \geq 0\}$ its positive cone, and by $\mathcal{E}(\mathcal{H})$ we denote the set of quantum effects, or simply, effects, that is, operators $A \in \mathcal{B}(\mathcal{H})$ such that $0 \leq A \leq I$. For two effects $A, B \in \mathcal{E}(\mathcal{H})$ we denote by $A \circ B=A^{1 / 2} B A^{1 / 2}$ the sequential product of the effects $A$ and $B$, that is, the effect obtained by doing the measurements in the order first $A$ and second $B$. Clearly, $A \circ B \leq A$ and a first question is when $A \circ B \leq B$. A sufficient condition to have this is the case when $A$ and $B$ are compatible, that is, $A B=B A$, but we would like to have a complete characterization. The answer is given by a particular case of the general majorization theorem (e.g. see [3]).

Theorem 2.1. Let $T_{i} \in \mathcal{B}\left(\mathcal{H}_{i}, \mathcal{G}\right)$ for Hilbert spaces $\mathcal{H}_{i}$ and $\mathcal{G}, i=1,2$. Then for $0 \leq \gamma<\infty$ the following conditions are equivalent:

(a) $\left\|T_{1}^{*} g\right\|_{\mathcal{H}_{1}} \leq \gamma\left\|T_{2}^{*} g\right\|_{\mathcal{H}_{2}}$ for all $g \in \mathcal{G}$;

(b) $T_{1} T_{1}^{*} \leq \gamma T_{2} T_{2}^{*}$;

(c) $T_{1}=T_{2} S$ for some operator $S \in \mathcal{B}\left(\mathcal{H}_{1}, \mathcal{H}_{2}\right)$ with $\|S\| \leq \gamma$.

Each of these equivalent conditions implies

(d) $\operatorname{Ran}\left(T_{1}\right) \subseteq \operatorname{Ran}\left(T_{2}\right)$.

Conversely, (d) implies all of (a) through (c), for some $\gamma<\infty$, the operator $S$ can be chosen such that $\operatorname{Ker}\left(T_{1}\right) \subseteq \operatorname{Ker}(S)$ and $\operatorname{Ker}\left(T_{2}\right) \subseteq \operatorname{Ker}\left(S^{*}\right)$, in which case it is uniquely determined.

As a consequence, given two operators $X, Y \in \mathcal{B}(\mathcal{H})$, we have $X X^{*} \leq Y Y^{*}$ if and only if $X=Y T$ for some $T \in \mathcal{B}(\mathcal{H}),\|T\| \leq 1$. In the following, for $A \in \mathcal{E}(\mathcal{H})$ we denote by $P_{A}$ the selfadjoint projection onto the closure of $\operatorname{Ran}(A)$ (which is the same with the closure of $\left.\operatorname{Ran}\left(A^{1 / 2}\right)\right)$.

Theorem 2.2. Let $A, B \in \mathcal{E}(\mathcal{H})$ be effects. Then $A \leq B$ if and only if there exists another effect $C \in \mathcal{E}(\mathcal{H})$ such that $A=B \circ C$. In addition, $C$ can be chosen such that $C \leq P_{A}$ and in this case it is uniquely determined.

Proof. Clearly, if $A=B \circ C$ for some effect $C \in \mathcal{E}(\mathcal{H})$, then $A=B \circ C \leq B$.

Conversely, let us assume that $A \leq B$. Then $A^{1 / 2} A^{1 / 2} \leq B^{1 / 2} B^{1 / 2}$ and hence, by Theorem 2.1. there exists $T \in \mathcal{B}(\mathcal{H})$, such that $\|T\| \leq 1$ and $A^{1 / 2}=B^{1 / 2} T$. Then $C=T T^{*} \in \mathcal{E}(\mathcal{H})$ and hence $A=B^{1 / 2} T T^{*} B^{1 / 2}=B \circ C$. The uniqueness part follows from the uniqueness part of Theorem 2.1

Corollary 2.3. Let $A, B \in \mathcal{E}(\mathcal{H})$. Then $A \circ B \leq B$ if and only if there exists $C \in \mathcal{E}(\mathcal{H})$ such that $A \circ B=B \circ C$. 
Corollary 2.3 says that the effect $A \circ B$ diminishes the effect $B$ if and only if there is another effect $C$ such that, performing first the measurement $B$ and then $C$, the obtained sequential measurement $B \circ C$ is the same as $A \circ B$.

In Theorem 3.2 in [5] it is proved that, given $A, B \in \mathcal{E}(\mathcal{H})$, we have the associativity property $(A \circ B) \circ C=A \circ(B \circ C)$, for all $C \in \mathcal{E}(\mathcal{H})$, if and only if $A$ and $B$ are compatible, that is, $A B=B A$. As a consequence of Theorem 2.2, we have:

Corollary 2.4. If $A, B, C \in \mathcal{E}(\mathcal{H})$ are effects, then there exists an effect $D \in \mathcal{E}(\mathcal{H})$ such that $A \circ(B \circ C)=(A \circ B) \circ D$.

Proof. Since $B \circ C \leq B$, we have $A \circ(B \circ C) \leq A \circ B$. Then by Theorem 2.2, there exists $D \in \mathcal{E}(\mathcal{H})$ such that $A \circ(B \circ C)=(A \circ B) \circ D$.

For a fixed $A \in \mathcal{E}(\mathcal{H})$, the mapping $\mathcal{E}(\mathcal{H}) \ni B \mapsto s_{A}(B)=A \circ B \in \mathcal{E}(\mathcal{H})$ is strongly continuous and affine. We are interested in sections of the sequential mapping $s_{A}$. Since $A \circ B \leq A$, it follows that such a section should be defined on $[0, A]=\{C \in \mathcal{E}(\mathcal{H}) \mid C \leq A\}$. Recall that $P_{A}$ denotes the selfadjoint projection onto the closure of $\operatorname{Ran}(A)$. By Theorem 2.2, for any $B \in[0, A]$ there exists a unique $C \in\left[0, P_{A}\right]$ such that $B=A \circ C$. Denote $B / A=C$ and call it the sequential quotient of $B$ by $A$. In this way, we can define a mapping

$$
f_{A}:[0, A] \rightarrow\left[0, P_{A}\right], \quad f_{A}(B)=B / A, B \in[0, A] .
$$

Clearly, $f_{A}$ is a section of $s_{A}$.

Let us also note that the "segment" $[0, A]$ has a natural sequential product, that we denote by $\times$,

$$
C \times D=A \circ((C / A) \circ(D / A)), \quad C, D \in[0, A] .
$$

Theorem 2.5. For any $A \in \mathcal{E}(\mathcal{H})$, the mapping $f_{A}$ defined as in (2.1) is an affine strongly continous homeomorphism, such that

$$
f_{A}(C \times D)=f_{A}(C) \circ f_{A}(D), \quad C, D \in[0, A] .
$$

Proof. Note first that $f_{A}$ is bijective: its inverse is $s_{A} \mid\left[0, P_{A}\right]:\left[0, P_{A}\right] \rightarrow[0, A]$. Thus, since $s_{A}$ is affine and $[0, A]$ is convex, $s_{A} \mid\left[0, P_{A}\right]$ is affine, hence $f_{A}$ is affine. To see that $f_{A}$ is strongly continuous, note that

$$
\langle B x, y\rangle=\left\langle(B / A) A^{1 / 2} x, A^{1 / 2} y\right\rangle, \quad B \in \mathcal{E}(\mathcal{H}), x, y \in \mathcal{H},
$$

and recall that the strong operator topology coincides with the weak operator topology on bounded sets of positive operators. Thus, $f_{A}$ is an affine strongly continuous homeomorphism. The property expressed in (2.3) is just another way of writing (2.2).

We are now interested in the question of when $A \circ B \geq B$. In Theorem 2.6 of [5] it is proved that, if $\mathcal{H}$ is finite dimensional, by preceding the effect $B$ with another effect $A$, we cannot amplify the effect $B$; more precisely, if $A \circ B \geq B$, then $A B=B A=B$, and it is asked whether this holds for infinite-dimensional spaces $\mathcal{H}$. In the following theorem we answer this question positively.

Theorem 2.6. Let $A, B \in \mathcal{E}(\mathcal{H})$ such that $A \circ B \geq B$. Then $A B=B A=B$ and, consequently, $A \circ B=B$. 
Proof. In the following we will repeatedly use the following elementary fact: if $C, D \in \mathcal{B}(\mathcal{H})$ are selfadjoint such that $C \leq D$, then for any $X \in \mathcal{B}(\mathcal{H})$ we have $X^{*} C X \leq X^{*} D X$.

Since $B \leq I$, it follows that $A^{1 / 2} B A^{1 / 2} \leq A^{1 / 2} A^{1 / 2}=A$. Therefore, by hypothesis we have

$$
0 \leq B \leq A^{1 / 2} B A^{1 / 2} \leq A \leq I .
$$

By applying $A^{1 / 2} \cdot A^{1 / 2}$ to (2.4), it follows that

$$
0 \leq A^{1 / 2} B A^{1 / 2} \leq A^{1 / 2} A^{1 / 2} B A^{1 / 2} A^{1 / 2}=A B A \leq A^{1 / 2} A A^{1 / 2}=A^{2} \leq A,
$$

and hence, using again (2.4), we have

$$
0 \leq B \leq A^{1 / 2} B A^{1 / 2} \leq A^{2 / 2} B A^{2 / 2} \leq A^{2} \leq A \leq I .
$$

Performing a similar procedure, we obtain inductively that for all $n \geq 1$ we have

$$
0 \leq B \leq A^{1 / 2} B A^{1 / 2} \leq \cdots \leq A^{n / 2} B A^{n / 2} \leq A^{n} \leq \cdots \leq A \leq I .
$$

From (2.5) we keep only

$$
0 \leq B \leq A^{n} \leq I, \quad \forall n \geq 1
$$

Let us now consider the sequence of functions $f_{n}(t)=t^{n}, f_{n}:[0,1] \rightarrow \mathbb{R}_{+}$. Note that $\left|f_{n}(t)\right| \leq 1$ for all $n \geq 1$ and that

$$
\lim _{n \rightarrow \infty} f_{n}(t)=\chi_{\{1\}}(t)= \begin{cases}1, & t=1, \\ 0, & 0 \leq t<1, \quad \forall t \in[0,1]\end{cases}
$$

that is, the sequence of functions $t^{n}$ converges boundedly pointwise, on the compact interval $[0,1] \supseteq \sigma(A)$, to the characteristic function of the set $\{1\}$. Thus, by borelian functional calculus for selfadjoint operators we have

$$
\text { so- } \lim _{n \rightarrow \infty} A^{n}=\text { so- } \lim _{n \rightarrow \infty} f_{n}(A)=\chi_{\{1\}}(A)=P_{\operatorname{Ker}(I-A)} .
$$

Consequently, letting $n \rightarrow \infty$ in (2.6), by (2.7) we obtain

$$
0 \leq B \leq P_{\operatorname{Ker}(I-A)} \text {. }
$$

If we represent the selfadjoint operators $A$ and $B$ with respect to the decomposition

$$
\mathcal{H}=\operatorname{Ker}(I-A) \oplus(\mathcal{H} \ominus \operatorname{Ker}(I-A)),
$$

by (2.8) we get

$$
A=\left[\begin{array}{cc}
I & 0 \\
0 & A_{22}
\end{array}\right], \quad B=\left[\begin{array}{cc}
B_{11} & 0 \\
0 & 0
\end{array}\right] .
$$

Now it is easy to see that $A B=B A=B$.

\section{Contractively contained Hilbert spaces}

Let $\mathcal{K}$ and $\mathcal{H}$ be Hilbert spaces. We say that $\mathcal{K}$ is continuously contained in $\mathcal{H}$, and we write $\mathcal{K} \hookrightarrow \mathcal{H}$, if $\mathcal{K} \subseteq \mathcal{H}$ and the inclusion mapping $\iota_{\mathcal{K}}: \mathcal{K} \hookrightarrow \mathcal{H}$ is continuous, that is, there exists $\gamma \geq 0$ such that $\|k\|_{\mathcal{H}} \leq \gamma\|k\|_{\mathcal{K}}$, for all $k \in \mathcal{K}$. $\mathcal{K}$ is contractively contained in $\mathcal{H}$ if $\mathcal{K} \subseteq \mathcal{H}$ and the inclusion mapping $\iota_{\mathcal{K}}: \mathcal{K} \hookrightarrow \mathcal{H}$ is contractive, that is, $\|k\|_{\mathcal{H}} \leq\|k\|_{\mathcal{K}}$, for all $k \in \mathcal{K}$.

Let $\mathcal{K} \hookrightarrow \mathcal{H}$ and denote $A=\iota_{\mathcal{K}} \iota_{\mathcal{K}}^{*} \in \mathcal{B}(\mathcal{H})$. Then $A \geq 0$ and, following L. Schwartz [8], we call it the kernel of $\mathcal{K}$ relative to $\mathcal{H}$. 
In the following we propose a geometric approach to quantum effects and their sequential product. Let $A \in \mathcal{B}(\mathcal{H}), A \geq 0$. Define $\mathcal{R}_{A}=\operatorname{Ran}\left(A^{1 / 2}\right)$ and the inner product

$$
\left\langle A^{1 / 2} x, A^{1 / 2} y\right\rangle_{\mathcal{R}_{A}}=\left\langle P_{\mathcal{H} \ominus \operatorname{Ker}(A)} x, y\right\rangle_{\mathcal{H}}, \quad x, y \in \mathcal{H} .
$$

More precisely, we define a Hilbert space structure on $\operatorname{Ran}\left(A^{1 / 2}\right)$ in such a way that the operator $A^{1 / 2}: \mathcal{H} \rightarrow \operatorname{Ran}\left(A^{1 / 2}\right)$ is a co-isometry. Thus, $\left(\mathcal{R}_{A},\langle\cdot, \cdot\rangle_{\mathcal{R}_{A}}\right)$ is a Hilbert space. We note that

$$
\left\|A^{1 / 2} x\right\|_{\mathcal{R}_{A}}=\left\|P_{\mathcal{H} \ominus \operatorname{Ker}(A)} x\right\|, \quad x \in \mathcal{H} .
$$

Then

$$
\begin{aligned}
\left\|A^{1 / 2} x\right\|_{\mathcal{H}} & =\left\|A^{1 / 2} P_{\mathcal{H} \ominus \operatorname{Ker}(A)} x\right\|_{\mathcal{H}} \leq\left\|A^{1 / 2}\right\| \cdot\left\|P_{\mathcal{H} \ominus \operatorname{Ker}(A)} x\right\| \\
& =\left\|A^{1 / 2}\right\| \cdot\left\|A^{1 / 2} x\right\|_{\mathcal{R}_{A}}, \quad x \in \mathcal{H} .
\end{aligned}
$$

Thus, $\mathcal{R}_{A} \hookrightarrow \mathcal{H}$ and $\left\|\iota_{\mathcal{R}_{A}}\right\| \leq\left\|A^{1 / 2}\right\|$; in particular, if $A \leq I$, then $\mathcal{R}_{A} \hookrightarrow \mathcal{H}$ contractively.

Let $x, y \in \mathcal{H}$ be arbitrary. Then

$$
\begin{aligned}
\left\langle\iota_{\mathcal{R}_{A}} y, A^{1 / 2} x\right\rangle_{\mathcal{R}_{A}} & =\left\langle y, A^{1 / 2} x\right\rangle_{\mathcal{H}}=\left\langle A^{1 / 2} y, x\right\rangle_{\mathcal{H}} \\
& =\left\langle P_{\mathcal{H} \ominus \operatorname{Ker}(A)} A^{1 / 2} y, x\right\rangle_{\mathcal{H}}=\left\langle A^{1 / 2} A^{1 / 2} y, A^{1 / 2} x\right\rangle_{\mathcal{R}_{A}},
\end{aligned}
$$

that is,

$$
\iota_{\mathcal{R}_{A}} y=A^{1 / 2} A^{1 / 2} y=P_{\mathcal{H} \ominus \operatorname{Ker}(A)} A y, \quad y \in \mathcal{H},
$$

and hence $\iota_{\mathcal{R}_{A}} \iota_{\mathcal{R}_{A}}^{*}=A$. Thus $A$ is the kernel of $\mathcal{R}_{A}$ relative to $\mathcal{H}$. In particular, if $\mathcal{R}_{A} \hookrightarrow \mathcal{H}$ contractively, then $A \leq I$.

Let $\mathcal{K}$ be another Hilbert space continuously contained in $\mathcal{H}$ and such that $\iota_{\mathcal{K}} \iota_{\mathcal{K}}^{*}=$ $A$. Then $\operatorname{Ran}(A)=\operatorname{Ran}\left(\iota_{\mathcal{K}}^{*}\right)$ is dense in $\mathcal{K}$. For arbitray $x, y \in \mathcal{H}$ we have

$$
\begin{aligned}
\langle A x, A y\rangle_{\mathcal{R}_{A}} & =\left\langle A^{1 / 2} x, A^{1 / 2} y\right\rangle_{\mathcal{H}}=\langle A x, y\rangle_{\mathcal{H}}=\left\langle\iota_{\mathcal{K}} \iota_{\mathcal{K}}^{*} x, y\right\rangle_{\mathcal{H}} \\
& =\left\langle\iota_{\mathcal{K}}^{*} x, \iota_{\mathcal{K}}^{*} y\right\rangle_{\mathcal{K}}=\langle A x, A y\rangle_{\mathcal{K}},
\end{aligned}
$$

that is, $\langle\cdot, \cdot\rangle_{\mathcal{R}_{A}}$ coincide with $\langle\cdot, \cdot\rangle_{\mathcal{K}}$ on a dense linear manifold, hence $\mathcal{R}_{A}=\mathcal{K}$ as Hilbert spaces.

Thus we have proved the following theorem, which is a particular case of results in [8] (see also [2] and [4]).

Theorem 3.1. Let $A \in \mathcal{B}(\mathcal{H})^{+}$. Then there exists uniquely a Hilbert space $\mathcal{R}_{A} \hookrightarrow$ $\mathcal{H}$ such that $A$ is the kernel of $\mathcal{R}_{A}$ relative to $\mathcal{H}$. In addition, $\mathcal{R}_{A} \hookrightarrow \mathcal{H}$ contractively if and only if $A \in \mathcal{E}(\mathcal{H})$.

Recall that for a Hilbert space $\mathcal{H}$ we denote $\mathcal{E}(\mathcal{H})=\{A \in \mathcal{B}(\mathcal{H}) \mid 0 \leq A \leq I\}$, identified with the set of quantum effects on $\mathcal{H}$, and by $\mathcal{P}(\mathcal{H})=\{P \in \mathcal{B}(\mathcal{H}) \mid P=$ $\left.P^{*}=P^{2}\right\}$ the set of orthogonal projections, identified with the set of sharp effects. As a consequence of Theorem 3.1, it follows that the mapping $A \mapsto \mathcal{R}_{A}$ induces a bijection between $\mathcal{E}(\mathcal{H})$ and the class of Hilbert spaces contractively contained in $\mathcal{H}$. This extends the bijective correspondence of $\mathcal{P}(\mathcal{H})$ with the class of subspaces of $\mathcal{H}$; a Hilbert space $\mathcal{K}$ contractively contained in $\mathcal{H}$ is a subspace of $\mathcal{H}$ if and only if the inclusion is isometric, and in this case $\iota_{\mathcal{K}} \iota_{\mathcal{K}}^{*}$ is the orthogonal projection of $\mathcal{H}$ onto $\mathcal{K}$. Note that, in this correspondence, subspace means that $\langle\cdot, \cdot\rangle_{\mathcal{K}}$ coincides with the restriction of $\langle\cdot, \cdot\rangle_{\mathcal{H}}$; if we impose only the condition that the strong topology 
of $\mathcal{K}$ is inherited from $\mathcal{H}$, we get the class of kernels with closed range. We make this precise in our context.

Corollary 3.2. Let $A, B \in \mathcal{B}(\mathcal{H})^{+}$. The following assertions are equivalent:

(i) $\mathcal{R}_{A} \subseteq \mathcal{R}_{B}$ (set inclusion).

(ii) $\mathcal{R}_{A} \hookrightarrow \mathcal{R}_{B}$.

(iii) There exists $\gamma \geq 0$ such that $A \leq \gamma B$.

In addition, denoting $\iota: \mathcal{R}_{A} \hookrightarrow \mathcal{R}_{B}$, the optimal constant $\gamma$ in (iii) is $\|\iota\|$. In particular, $\mathcal{R}_{A} \hookrightarrow \mathcal{R}_{B}$ contractively if and only if $A \leq B$.

Proof. Consequence of Theorem 2.1 and Theorem 3.1

Corollary 3.3. Let $A, B \in \mathcal{B}(\mathcal{H})^{+}$. The following assertions are equivalent:

(i) $\mathcal{R}_{A}=\mathcal{R}_{B}$ (equality of sets).

(ii) $\mathcal{R}_{A}=\mathcal{R}_{B}$ (equality of sets) and the norms are equivalent.

(iii) There exists $\gamma>0$ such that $\frac{1}{\gamma} B \leq A \leq \gamma B$.

As a consequence of Corollary 3.3 , two Hilbert spaces $\mathcal{H}_{1}$ and $\mathcal{H}_{2}$, continuously contained in the Hilbert space $\mathcal{H}$, coincide as sets if and only if they coincide topologically, that is, their strong topologies coincide.

On the grounds of Theorem 3.1 we can define an operation of sequential prod$u c t$ for contractively contained Hilbert spaces. Let $\mathcal{H}_{1}$ and $\mathcal{H}_{2}$ be two Hilbert spaces contractively contained in $\mathcal{H}$, and let $A, B \in \mathcal{E}(\mathcal{H})$ be their kernels, that is, $\mathcal{H}_{1}=\mathcal{R}_{A}$ and $\mathcal{H}_{2}=\mathcal{R}_{B}$. Then $A \circ B=A^{1 / 2} B A^{1 / 2} \in \mathcal{E}(\mathcal{H})$ and we define $\mathcal{H}_{1} \circ \mathcal{H}_{2}=\mathcal{R}_{A \circ B} \hookrightarrow \mathcal{H}$ contractively. Of course, this definition can be extended for continuously contained Hilbert spaces, but in this more general case we have only that $\mathcal{H}_{1} \circ \mathcal{H}_{2}$ is continuosly contained in $\mathcal{H}$, unless $\mathcal{H}_{1}$ is contractively contained in $\mathcal{H}$. The next corollary says that the sequential product is natural for the study of contractively contained Hilbert spaces (compare with Theorem 2.2).

Corollary 3.4. Let $\mathcal{H}_{1} \hookrightarrow \mathcal{H}$ contractively. Then, for any Hilbert space $\mathcal{H}_{2} \hookrightarrow \mathcal{H}_{1}$ contractively, there exists $\mathcal{H}_{3} \hookrightarrow \mathcal{H}$ contractively, such that $\mathcal{H}_{2}=\mathcal{H}_{1} \circ \mathcal{H}_{3}$.

Proof. Consequence of Theorem 2.2 and Corollary 3.2

Another consequence of Theorem 3.1 is that we can define a "sequential quotient" for contractively contained Hilbert spaces as the "inverse" of the operation in Corollary 3.4 if the Hilbert space $\mathcal{H}_{2} \hookrightarrow \mathcal{H}_{1}$ contractively, then $\mathcal{H}_{2} / \mathcal{H}_{1}$ is the unique Hilbert space $\mathcal{H}_{3} \hookrightarrow \mathcal{H}$ contractively, such that $\mathcal{H}_{2}=\mathcal{H}_{1} \circ \mathcal{H}_{3}$.

Now let $\mathcal{K}$ be a Hilbert space contractively contained in $\mathcal{H}$. For any Hilbert spaces $\mathcal{H}_{1}$ and $\mathcal{H}_{2}$ contractively contained in $\mathcal{K}$, a natural "sequential product" denoted by $\times$ can be defined as

$$
\mathcal{H}_{1} \times \mathcal{H}_{2}=\mathcal{K} \circ\left(\left(\mathcal{H}_{1} / \mathcal{K}\right) \circ\left(\mathcal{H}_{2} / \mathcal{K}\right)\right),
$$

by analogy with (2.2). Then the sequential quotient is left distributive with respect to these sequential products, which is just another way of writing (3.2), by analogy with (2.3).

Corollary 3.5. Let $\mathcal{K}$ be a Hilbert space contractively contained in $\mathcal{H}$. For any Hilbert spaces $\mathcal{H}_{1}$ and $\mathcal{H}_{2}$ contractively contained in $\mathcal{K}$, we have

$$
\left(\mathcal{H}_{1} \times \mathcal{H}_{2}\right) / \mathcal{K}=\left(\mathcal{H}_{1} / \mathcal{K}\right) \circ\left(\mathcal{H}_{2} / \mathcal{K}\right) .
$$


The geometric approach of quantum effects allows us to consider the lattice properties of $\mathcal{E}(\mathcal{H})$, especially those in connection with "addition" and "intersection" of continuously contained Hilbert spaces. The addition of continously contained Hilbert spaces should correspond to addition of the kernels; cf. [8]. Here is a direct argument; cf. 4].

Let $A, B \in \mathcal{B}(\mathcal{H})^{+}$be such that $\mathcal{H}_{1}=\mathcal{R}_{A}$ and $\mathcal{H}_{2}=\mathcal{R}_{B}$. We consider the bounded operator $T=\left[\begin{array}{ll}A^{1 / 2} & -B^{1 / 2}\end{array}\right] \in \mathcal{B}(\mathcal{H} \oplus \mathcal{H}, \mathcal{H})$. Then

$$
\begin{aligned}
\mathcal{R}_{A}+\mathcal{R}_{B} & =\operatorname{Ran}\left(A^{1 / 2}\right)+\operatorname{Ran}\left(B^{1 / 2}\right)=\operatorname{Ran}(T)=\operatorname{Ran}\left(\left(T T^{*}\right)^{1 / 2}\right) \\
& =\operatorname{Ran}\left((A+B)^{1 / 2}\right)=\mathcal{R}_{A+B},
\end{aligned}
$$

where we took into account that, by polar decomposition, $T=\left|T^{*}\right| V$, where $\left|T^{*}\right|=$ $\left(T T^{*}\right)^{1 / 2}$ and $V$ is a partial isometry. Consequently, we have

Theorem 3.6 (e.g. see [8] and [4]). Let $\mathcal{H}_{1}$ and $\mathcal{H}_{2}$ be two Hilbert spaces continuously contained in $\mathcal{H}$. Then the algebraic sum $\mathcal{H}_{1}+\mathcal{H}_{2}$ can be naturally organized as a Hilbert space continuously contained in $\mathcal{H}$; more precisely, if $A, B \in \mathcal{B}(\mathcal{H})^{+}$ are the kernels of $\mathcal{H}_{1}$ and respectively $\mathcal{H}_{2}$, that is, $\mathcal{H}_{1}=\mathcal{R}_{A}$ and $\mathcal{H}_{2}=\mathcal{R}_{B}$, then $\mathcal{R}_{A}+\mathcal{R}_{B}=\mathcal{R}_{A+B}$ as sets.

The intersection of continuously contained Hilbert spaces is a much more subtle operation that requires the definition of parallel sum of kernels, as introduced for the finite-dimenensional case in [1], and in general in [4] (cf. [7] for a further study). Let $A, B \in \mathcal{B}(\mathcal{H})^{+}$. Since $A \leq A+B$, by Theorem 2.1 there exists a unique contraction $X \in \mathcal{B}(\mathcal{H})$ such that

$$
A^{1 / 2}=(A+B)^{1 / 2} X, \quad \operatorname{Ker}\left(X^{*}\right) \supseteq \operatorname{Ker}(A+B) .
$$

Similarly, since $B \leq A+B$, there exists a unique contraction $Y \in \mathcal{B}(\mathcal{H})$ such that

$$
B^{1 / 2}=(A+B)^{1 / 2} Y, \quad \operatorname{Ker}\left(Y^{*}\right) \supseteq \operatorname{Ker}(A+B) .
$$

The parallel sum of $A$ and $B$ is defined as

$$
A: B=A^{1 / 2} X^{*} Y B^{1 / 2} \text {. }
$$

Theorem $3.7\left([4]\right.$ and [7])). Let $A, B \in \mathcal{B}(\mathcal{H})^{+}$. Then:

(i) $0 \leq A: B \leq A, B$.

(ii) $A: B=B: A$.

(iii) $\operatorname{Ran}\left((A: B)^{1 / 2}\right)=\operatorname{Ran}\left(A^{1 / 2}\right) \cap \operatorname{Ran}\left(B^{1 / 2}\right)$.

(iv) If $A_{1}, B_{1} \in \mathcal{B}(\mathcal{H})^{+}$are such that $A \leq A_{1}$ and $B \leq B_{1}$, then $A$ : $B \leq A_{1}: B_{1}$.

(v) If $A, B \neq 0$, then $\|A: B\| \leq\left(\|A\|^{-1}+\|B\|^{-1}\right)^{-1}$.

(vi) $\langle(A: B) h, h\rangle=\inf \{\langle A a, a\rangle+\langle B b, b\rangle \mid h=a+b\}$, for all $h \in \mathcal{H}$.

(vii) If $A_{n} \searrow A$ and $B_{n} \searrow B$ strongly, then $A_{n}: B_{n} \searrow A: B$ strongly.

According to (iii) we can give an interpretation of the binary operation of "intersection" for continuously contained Hilbert spaces as follows:

Theorem 3.8. Let $\mathcal{H}_{1}$ and $\mathcal{H}_{2}$ be two Hilbert spaces continuously contained in the Hilbert space $\mathcal{H}$. Then the vector space $\mathcal{H}_{1} \cap \mathcal{H}_{2}$ can be naturally organized as a Hilbert space continuously contained in $\mathcal{H}$; more precisely, letting $\mathcal{H}_{1}=\mathcal{R}_{A}$ and $\mathcal{H}_{2}=\mathcal{R}_{2}$ for $A, B \in \mathcal{B}(\mathcal{H})^{+}$, identify $\mathcal{H}_{1} \cap \mathcal{H}_{2}$ with $\mathcal{R}_{A: B}$. If either of $\mathcal{H}_{i}, i=1,2$, is contractively contained in $\mathcal{H}$, that is, its kernel is a quantum effect, then $\mathcal{H}_{1} \cap \mathcal{H}_{2}$ is contractively contained in $\mathcal{H}$. 
Another simple consequence of Theorem 3.7 is the stability of the set of quantum effects under the operation of parallel sum, even with the factor 2.

Corollary 3.9. Let $A, B \in \mathcal{E}(\mathcal{H})$. Then $2(A: B) \in \mathcal{E}(\mathcal{H})$.

Proof. By Theorem 3.7(i), we have $A: B \geq 0$, so it remains to prove that $2(A: B) \leq$ $I$. To see this we use Theorem $3.7(\mathrm{v})$ and the inequality between the harmonic mean and the geometric mean to see that

$$
\|2(A: B)\| \leq \frac{2\|A\|\|B\|}{\|A\|+\|B\|} \leq \sqrt{\|A\|\|B\|} \leq 1,
$$

and hence $2(A: B) \leq I$.

We end by proving that the sequential product is a morphism, in the second variable, with respect to the parallel sum. Recall that for any $A \in \mathcal{E}(\mathcal{H})$ we denote $[0, A]=\{B \mid 0 \leq B \leq A\}$ and $P_{A}$ is the orthogonal projection onto the closure of $\operatorname{Ran}(A)$.

Theorem 3.10. Let $A \in \mathcal{E}(\mathcal{H})$ and consider the mapping $s_{A}:\left[0, P_{A}\right] \rightarrow[0, A]$, $s_{A}(B)=A \circ B=A^{1 / 2} B A^{1 / 2}$, for all $B \in\left[0, P_{A}\right]$. Then

$$
s_{A}(C: D)=s_{A}(C): s_{A}(D), \quad C, D \in\left[0, P_{A}\right] .
$$

Proof. Let $C, D \in\left[0, P_{A}\right]$ and consider the operators $X, Y$ uniquely determined such that

$$
\begin{array}{ll}
C^{1 / 2}=(C+D)^{1 / 2} X, & \operatorname{Ker}\left(C^{*}\right) \supseteq \operatorname{Ker}(C+D), \\
D^{1 / 2}=(C+D)^{1 / 2} Y, & \operatorname{Ker}\left(D^{*}\right) \supseteq \operatorname{Ker}(C+D) .
\end{array}
$$

Then, by definition,

$$
C: D=C^{1 / 2} X^{*} Y D^{1 / 2}, \quad s_{A}(C: D)=A^{1 / 2} C^{1 / 2} X^{*} Y D^{1 / 2} A^{1 / 2} .
$$

Since $s_{A}(C)+s_{A}(D)=A^{1 / 2}(C+D) A^{1 / 2}$, it follows that there exists a uniquely determined partial isometry $V$, with appropriate supports, such that

$$
\left(s_{A}(C)+s_{A}(D)\right)^{1 / 2} V=A^{1 / 2}(C+D)^{1 / 2} .
$$

Similarly, there exist uniquely determined partial isometries $U$ and $W$, with appropriate supports, such that

$$
s_{A}(C)^{1 / 2}=A^{1 / 2} C^{1 / 2} U, \quad s_{A}(D)^{1 / 2}=A^{1 / 2} D^{1 / 2} W .
$$

Therefore,

$$
\begin{gathered}
s_{A}(C)^{1 / 2}=A^{1 / 2} C^{1 / 2} U=A^{1 / 2}(C+D)^{1 / 2} X U=\left(s_{A}(C)+s_{A}(D)\right)^{1 / 2} V X U, \\
s_{A}(D)^{1 / 2}=A^{1 / 2} D^{1 / 2} U=A^{1 / 2}(C+D)^{1 / 2} X W=\left(s_{A}(C)+s_{A}(D)\right)^{1 / 2} V X W,
\end{gathered}
$$

and hence, by the definition of the parallel sum and taking into account that since $C$ and $D$ are in $\left[0, P_{A}\right]$, it follows that multiplication on the left with $A^{1 / 2}$ does not affect the kernels, we have

$$
\begin{aligned}
s_{A}(C): s_{A}(D) & =s_{A}(C)^{1 / 2} U^{*} X^{*} V^{*} V Y W^{*} s_{A}(D)^{1 / 2} \\
& =A^{1 / 2} C^{1 / 2} X^{*} Y D^{1 / 2} A^{1 / 2}=s_{A}(C: D) .
\end{aligned}
$$


With the definition of "intersection" of continuously contained Hilbert spaces, as in Theorem 3.8, and the definition of "sequential product" for contractively contained Hilbert spaces as a consequence of Theorem [3.1, Theorem [3.10] can be rephrased to say that the sequential product is left distributive with respect to the intersection.

Theorem 3.11. Let the Hilbert space $\mathcal{H}_{0}$ be contractively contained in the Hilbert space $\mathcal{H}$. Then, for any Hilbert spaces $\mathcal{K}$ and $\mathcal{G}$, contractively contained in $\overline{\mathcal{H}_{0}}$, the closure of $\mathcal{H}_{0}$ in $\mathcal{H}$, we have

$$
\mathcal{H}_{0} \circ(\mathcal{K} \cap \mathcal{G})=\left(\mathcal{H}_{0} \circ \mathcal{K}\right) \cap\left(\mathcal{H}_{0} \circ \mathcal{G}\right) .
$$

Remark 3.12. Alternatively, Theorem 3.10 can be proved by Theorem 3.7(vi). Thus, for any quantum effects $0 \leq C, D \leq P_{A}$ and $h \in \mathcal{H}$ we have

$$
\left\langle s_{A}(C: D) h, h\right\rangle=\inf \left\{\langle C x, x\rangle+\langle D y, y\rangle \mid A^{1 / 2} h=x+y\right\} .
$$

Both $C$ and $D$ have supports in $P_{A} \mathcal{H}=\overline{\operatorname{Ran}\left(A^{1 / 2}\right)}$ and hence

$$
\begin{aligned}
\left\langle s_{A}(C\right. & : D)) h, h\rangle \\
& =\inf \left\{\left\langle C A^{1 / 2} c, A^{1 / 2} c\right\rangle+\left\langle D A^{1 / 2} d, A^{1 / 2} d\right\rangle \mid A^{1 / 2} h=A^{1 / 2} c+A^{1 / 2} d\right\} \\
& =\inf \left\{\left\langle A^{1 / 2} C A^{1 / 2} c, c\right\rangle+\left\langle A^{1 / 2} D A^{1 / 2} d, d\right\rangle \mid h=c+d\right\} \\
& =\left\langle s_{A}(C): s_{A}(D) h, h\right\rangle,
\end{aligned}
$$

where we take into account that, without restricting the generality, we can take all the vectors $h, c$, and $d$ in $P_{A} \mathcal{H}$ and that on this subspace $A^{1 / 2}$ is one-to-one.

Corollary 3.13. Let $A \in \mathcal{E}(\mathcal{H})$ and consider the function $f_{A}$ as defined in (2.1). Then,

$$
f_{A}(E: F)=f_{A}(E): f_{A}(F), \quad E, F \in[0, A] .
$$

Proof. The function $f_{A}$ is the inverse of the function $s_{A}$ as in Theorem 3.10 ,

This corollary can be rephrased, in terms of the "sequential quotient" as defined in (3.2), saying that the "sequential product" of contractively contained Hilbert spaces is right distributive with respect to "intersection".

Corollary 3.14. Let $\mathcal{K}$ be a Hilbert space contractively contained in $\mathcal{H}$. For any Hilbert spaces $\mathcal{H}_{1}$ and $\mathcal{H}_{2}$ contractively contained in $\mathcal{K}$, we have

$$
\left(\mathcal{H}_{1} \cap \mathcal{H}_{2}\right) / \mathcal{K}=\left(\mathcal{H}_{1} / \mathcal{K}\right) \cap\left(\mathcal{H}_{2} / \mathcal{K}\right) .
$$

\section{REFERENCES}

[1] W. N. Anderson, Jr. and R. J. Duffin: Series and parallel addition of matrices, J. Math. Anal. Appl., 26 (1969), 576-594. MR 39:3904

[2] L. de Branges and J. Rovnyak: Canonical models in quantum scattering theory, in Perturbation Theory and its Applications in Quantum Mechanics (Proc. Adv. Sem. Math. Res. Center, U. S. Army, Theoret. Chem. Inst., Univ. of Wisconsin, Madison, Wis., 1965) pp. 295-392, Wiley, New York, 1966. MR 39:6109

[3] R. G. Douglas: On majorization, factorization, and range inclusion of operators on Hilbert space, Proc. Amer. Math. Soc., 17 (1966), 413-415. MR 34:3315

[4] P. A. Fillmore and J. P. Williams: On operator ranges, Advances in Math., 7 (1971), 254-281. MR 45:2518

[5] S. Gudder and G. Nagy: Sequential quantum measurements, J. Math. Phys., 42 (2001), 5212-5222. MR 2002h:81032 
[6] S. Gudder and G. Nagy: Sequentially independent effects, Proc. Amer. Math. Soc., 130 (2002), 1125-1130. MR 2002i:81014

[7] E. L. Pekarev and Yu. L. Shmulyan: Parallel addition and parallel subtraction of operators [Russian], Izv. Akad. Nauk SSSR, Ser. Mat., 40 (1976), 366-387. MR 53:14178

[8] L. Schwartz: Sous-espaces Hilbertiens d'espaces vectoriel topologiques et noyaux associés (noyaux reproduisants), J. Analyse Math., 13 (1964), 115-256. MR 31:3835

Institutul de Matematică al ACademiei Române, C.P. 1-764, 014700 Bucureşti, ROMÂNIA

E-mail address: gheondea@theta.ro

Current address: Department of Mathematics, Bilkent University, 06533 Ankara, Turkey

Department of Mathematics, University of Denver, Denver, Colorado 80208

E-mail address: sgudder@math.du.edu 